\title{
Probing for stomach using the Focused Impedance Method (FIM)
}

\author{
Rashida Haque ${ }^{1}$, Muhammad Abdul Kadir ${ }^{1}$ and K Siddique-e Rabbani ${ }^{1,2}$ \\ 1. Department of Biomedical Physics and Technology, University of Dhaka, Dhaka 1000, Bangladesh \\ 2. E-mail any correspondence to: rabbani@du.ac.bd
}

\begin{abstract}
For probing deep organs of the body using electrical impedance, the conventional method is to use Electrical Impedance Tomography (EIT). However, this would be a sophisticated machine and will be very expensive when a full 3D EIT is developed in the future. Furthermore, for most low income countries such expensive devices may not deliver the benefits to a large number of people. Therefore, this paper suggests the use of simpler techniques like Tetrapolar Impedance Measurement (TPIM) or Focused Impedance Method (FIM) in probing deeper organs. Following a method suggested earlier by one of the authors, this paper studies the possibility of using TPIM and FIM for the stomach. Using a simplified model of the human trunk with an embedded stomach, a finite element simulation package, COMSOL, was used to obtain transfer impedance values and percentage contribution of the stomach region in the total impedance. For this work, judicious placement of electrodes through qualitative visualizations based on point sensitivity equations and equipotential concepts were made, which showed that reasonable contribution of the stomach region is possible through the use of TPIM and FIM. The contributions were a little over $20 \%$ which is of similar order of the cross-sectional area percentage of the stomach with respect to that of the trunk. For the case where the conductivity of the stomach region was assumed about 4 times higher, the contributions increased to about $38 \%$. Through further studies this proposed methods may contribute greatly in the study of deeper organs of the body.
\end{abstract}

Keywords: Electrical bio-impedance; deep organ bio-impedance; EIT; TPIM; FIM

\section{Introduction}

Historically, using electrical impedance of the human body for physiological study or diagnosis flourished after the introduction of the Tetrapolar Impedance Measurement (TPIM) technique by Nyboer [1], eliminating the skin- electrode contact impedance, which is very large compared to that of the bulk at typical measurement frequencies that had posed a challenge before. In this method, since the current drive and potential measuring electrodes are different, the value obtained is strictly not impedance, rather it is a "transfer Impedance" [2]. In a volume conductor, the transfer impedance very much depends on the geometry of the conductor, placement of the electrodes and the conductivity distribution inside. Therefore, different transfer impedance values will be obtained for different electrode placements, having more sensitivity to certain regions of the volume. TPIM has poor geometrical localization in terms of sensitivity and was used mainly for whole body measurements $[3,4]$.

A distribution image of impedance within a volume conductor named, "Electrical Impedance Tomography (EIT)" became popular after the pioneering works by several groups $[5,6]$. The technique uses multiple TPIM measurements using 16, 32 or more electrodes fixed around a volume conductor and was initially proposed for obtaining 2D tomographic images. This is a complex technique, requiring sophisticated hardware and computer algorithms. Since electrical current spreads out in all directions and does not follow a straight path, 2D images in fact contained significant contributions from 3D [7].

Eventually EIT was extended for 3D imaging using multiple level electrode arrays and is still going through an R\&D phase [8]. Obviously, the technology, when ready, would be more complex, contributing to a high cost and sophistication, and may find little use in low and medium income countries (LMIC), which is an area of interest of the present authors. 
The authors' extended group at Dhaka University introduced the Focused Impedance Method (FIM) as a bridge between TPIM and EIT [9-11]. Using 8, 6 or 4 electrodes it can localize a target region [12]. The complexity is much less than that of EIT and is marginally greater than that of TPIM. FIM has shown success in localized lung ventilation and gastric emptying, giving similar results as obtained using 2D EIT $[9,13]$. However, most of the applications attempted so far using FIM targeted measurement from one side of the human body in which contribution of the deep organs were limited, only partial contribution were coming from the regions at shallow depths with respect to the electrodes. In order to probe deeper regions of these

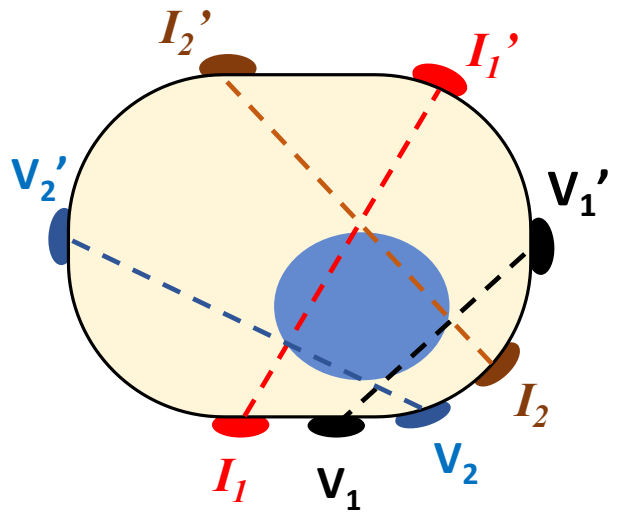

higher near to the bottom of the diagram, since the electrode separation between the respective I and $\mathrm{V}$ electrodes are less on this side of the volume conductor. The right hand diagram indicates that the target object, falling within the four equipotential lines, will have an increased contribution to the overall FIM value, measured using this configuration of electrodes. Of course, this configuration is just a qualitative suggestion, simulation using a finite element method (FEM) will give a more optimized configuration.

Based on the above suggestions, this work carries out a detailed FEM simulation using the COMSOL software package [18] on a simplified model of the human trunk and

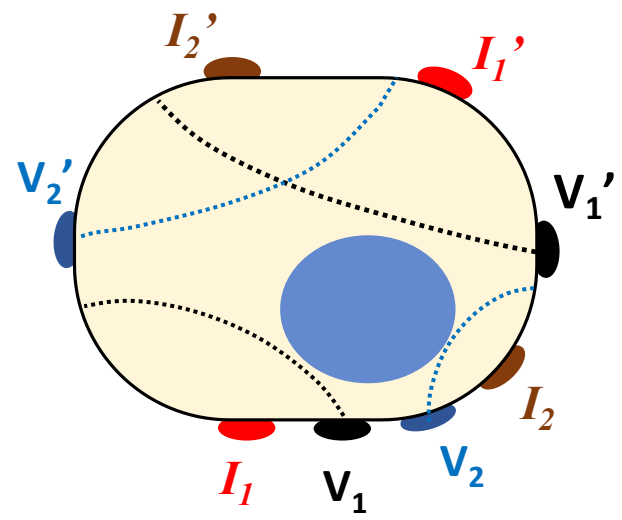

Fig 1: 8-electrode FIM to localise a deep target object in a volume conductor. Two pairs of electrodes indicated by $I_{1}-I_{1}{ }^{\prime}$ and $V_{1}-V_{1}{ }^{\prime}$ form the first TPIM configuration while $I_{2}-I_{2}^{\prime}$ and $V_{2}-V_{2}{ }^{\prime}$ indicate the second TPIM. The average of the two TPIM values make the FIM [14]. The dashed lines in the left hand figure show the maximum current lines between respective pairs of electrodes, which also includes the potential measuring electrodes, while the dotted lines on the right hand figure show approximate equipotential lines through the potential measuring electrodes.

organs, some new ideas on the placements of electrodes were suggested [14] using visualizations based on the concepts of equipotential surfaces [15] and of sensitivity equations involving current density vectors due to two separate pairs of electrodes in TPIM [16]. Finite element simulation and phantom measurements using one of these suggested methods targeting the lungs produced promising results [17]. The present work was taken up using another of the novel ideas on electrode placement suggested in the above work [14] that has the possibility of targeting the stomach. This idea was originally suggested for an asymmetrically placed deep object as shown in a cross sectional diagram in Figure 1. Here, two pairs of electrodes indicated by $I_{1}-I_{1}{ }^{\prime}$ and $V_{1}-V_{1}{ }^{\prime}$ form the first TPIM configuration while $I_{2}-I_{2}^{\prime}$ and $V_{2}-V_{2}{ }^{\prime}$ indicate the second. The algebraic average of the two TPIM values make the FIM. The dashed lines in the left hand figure show the maximum current lines between respective pairs of electrodes, which also includes the potential measuring electrodes, while the dotted lines on the right hand figure show approximate equipotential lines through the potential measuring electrodes. The left hand diagram suggests that the region between the maximum current lines will have high sensitivity to the overall transfer impedances through both the TPIM configurations, being the stomach and several electrode configurations, choosing the electrode configurations rather intuitively. Through this, the present work attempts to determine the contribution of the modeled stomach in the measurement of the total transfer impedance to find out if the suggested idea provides outcomes as anticipated. Then changing the electrode configurations a little from the starting configuration, this work will try to find the optimum electrode positions to get a maximum contribution. It will also explore if a simple TPIM with a suitable electrode placement may provide contribution figures close to that from FIM. Furthermore, based on these results, it will try to explore if some more novel electrode configurations may be more effective for the stomach.

\section{Materials and methods}

As mentioned above, the present work intends to get a reasonably high contribution from the stomach within a human trunk. Following the method suggested above, this measurement will be performed on a transverse plane of the trunk at the level of the stomach. Placing electrodes suitably to opposite sides of this cross-sectional plane it is expected that a reasonably high contribution from the stomach will be obtained in the total transfer impedance measurement. 


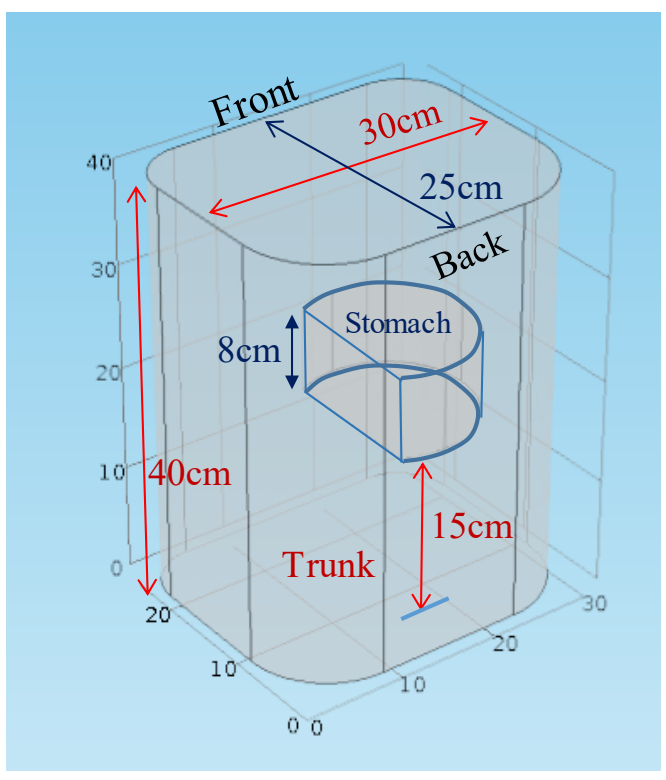

Fig 2: The simplified model for human trunk and an embedded stomach as used in the simulation. Some relevant dimensions are shown.

As a first attempt at simulation for the stomach, the present work chose a very simplified model of the human trunk with an embedded simplified stomach model as shown in Figure 2. The trunk was simulated by a vertical rectangular parallelepiped with rounded edges on all four sides. The horizontal dimensions chosen were $30 \mathrm{~cm}$ (representing trunk breadth, $x$-axis) and $25 \mathrm{~cm}$ (representing trunk thickness or depth, y-axis) while the vertical dimension was chosen at $40 \mathrm{~cm}$ (representing trunk height, z-axis). The radii of curvature for rounding of the rectangle at the corners were made greater on the assumed back of the body compared to that for the front; the values arbitrarily chosen at $8 \mathrm{~cm}$ and $5 \mathrm{~cm}$ respectively. This was done since the human trunk has similar features. Figure 3 shows a cross section of the trunk with the stomach model embedded.

In order to model the simplified stomach, a CT scan [19] was used as a guide. For this simulation, the stomach was modeled as a truncated circular cylinder with diameter 16 $\mathrm{cm}$ and height $8 \mathrm{~cm}$ (Figures 2 and 3). The cylinder was truncated vertically on the right side, $4 \mathrm{~cm}$ from the edge of the circle, making the lateral dimension of the modeled stomach as $12 \mathrm{~cm}$. The lower edge of the modeled stomach was placed at a height of $15 \mathrm{~cm}$ from the bottom of the trunk (Figure 2). It was placed towards the left side of the trunk, its left edge placed at a distance of $3 \mathrm{~cm}$ from the left trunk surface and the front edge placed at a distance of $3 \mathrm{~cm}$ from the front trunk surface as shown in Figure 3.

For the simulation, the conductivity $\sigma$ and the relative permittivity $\varepsilon$ of the volume conductor were chosen as appropriate for isotonic $0.9 \%$ saline [20], while those for the stomach were arbitrarily chosen, of a higher conductivity. Here, these were chosen as that for seawater, (3.5\% saline) [21]. This work was just to determine the contribution of the stomach region in the overall measurements, so was not related to real human tissues or real parameters for any food.

Electrodes were initially modeled as circular cylinders with a cross sectional diameter of $2 \mathrm{~cm}$ and length $3 \mathrm{~cm}$ each, but embedded along the boundary of the volume conductor such that the portion outside the surface of the trunk has a high conductivity, that for a metal, while the portion inside the surface, within the volume conductor, equals that for the volume conductor. Effectively, the electrodes just touched the outer surface of the volume conductor. Since on moving the conductor at different sites on the periphery changed the part of the electrode inside and outside the trunk volume, this feature was selected in COMSOL through appropriate partitioning of the electrode volume.

The placement of the electrodes chosen for the initial simulation are shown in Figure 4. Here, $I_{1} \& I_{1}^{\prime}$ represent the current drive electrodes while $V_{1} \& V_{1}^{\prime}$ represent the potential electrodes of a TPIM configuration (say, TPIM1). Similarly, $I_{2} \& I_{2}^{\prime}$ and $V_{2} \& V_{2}^{\prime}$ indicate electrodes for the second TPIM (say, TPIM2). These two TPIMs have been chosen almost perpendicular to each other expecting the sensitive zones to cross over the stomach region, increasing the overall sensitivity there. The logic for choosing a smaller separation for $I_{1}-V_{1}$ compared to $I_{1}^{\prime}-V_{1}^{\prime}$ in TPIM1 is that one may expect a higher sensitivity near to $I_{1}-V_{1}$ (around the stomach) compared to that near $I_{1}^{\prime}-V_{1}^{\prime}$ as mentioned before. The same arguments hold for the electrode separations in TPIM2.

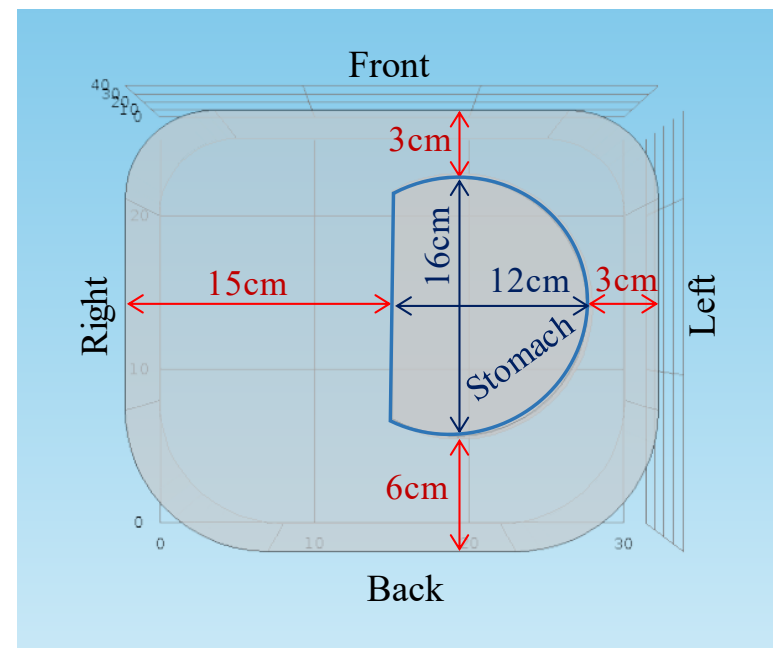

Fig 3: Cross sectional view of the trunk and the embedded stomach model as used in the simulation. The dimensions and position of the stomach are shown. The right and left side of the trunk are also indicated.

The above logic is the result of a visualization of the equation for conventional point sensitivity $S$ in transfer impedance in a TPIM [16, 22], assuming unit current densities,

$$
\begin{aligned}
S & =\frac{J_{1} \cdot J_{2}}{I^{2}} \\
\text { Or, } S & =\frac{J_{1} J_{2} \cos \theta}{I^{2}}
\end{aligned}
$$


where $\boldsymbol{J}_{\mathbf{1}}$ and $\boldsymbol{J}_{\mathbf{2}}$ represent the current density vectors at the point in question due to current driven through the two pairs of electrodes, both through the current drive electrodes and through the potential electrodes, $l$ is the magnitude of the current (equal through both pairs) and $\theta$ is the angle between the two vectors.

One can visualize that $S$ will be high if both the magnitudes, $J_{1}$ and $J_{2}$ are high and are in the same direction $\left(\theta=0^{\circ}\right)$, or have a small angle between them. $S$ will be low if $J_{1}$ and $J_{2}$ have very low values both, or are nearly perpendicular to each other $\left(\theta=90^{\circ}\right)$. If the angle is greater than $90^{\circ}$ than the sensitivity will be negative. The dashed straight lines between specific pairs of electrodes in Figure 4 show the current lines with the highest current density values for currents through the respective pairs, as these are the shortest current paths. This of course assumes a uniform conductivity throughout the volume conductor. The current lines will be curved away on both sides of the dashed straight lines having longer paths, so the current density values will decrease. The positions of the electrodes and the boundaries of the volume conductor will also influence the curvatures and path lengths of the current lines, resulting in further modifications of the current density vectors. Therefore, in between the dashed lines shown for TPIM1 (between $I_{1} \& I_{1}{ }^{\prime}$ and $\left.V_{1} \& V_{1}^{\prime}\right)$, the current lines will be squeezed in the middle, so the density will be high within the target object. Again, since the separation between $l_{1} \& V_{1}$ is less than that between $I_{1}^{\prime} \& V_{1}^{\prime}$, the current lines will be more squeezed near the top electrodes in Figure 4 and less near the bottom. This is expected to give a higher sensitivity in the top part of the volume conductor, where the object (stomach) is. Through a similar logic, the adjacent electrodes of TPIM2 have been made closer on the right side of the diagram (left side of the body) and wider in the opposite direction.

COMSOL simulation was made to obtain TPIM1 and TPIM2 and averaging them to obtain FIM for the two configurations as mentioned above. In each case the total

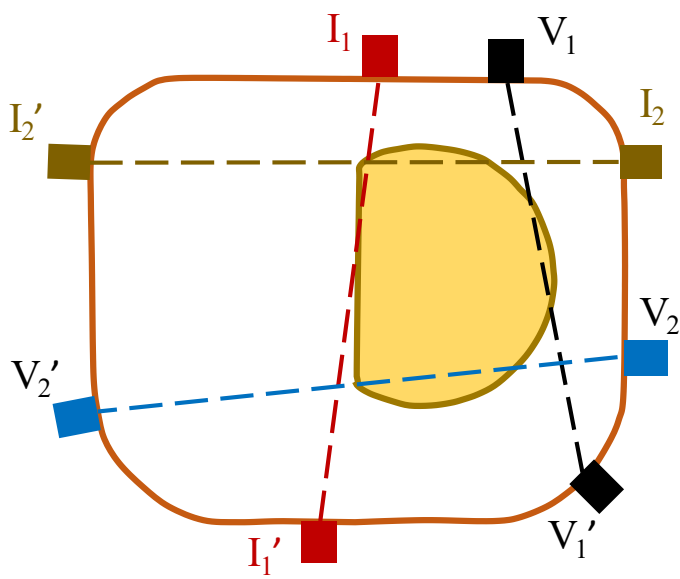

Fig 4: Cross sectional view showing the initial suggested positions of electrodes for the trunk and the embedded stomach model. The dashed straight lines represent the maximum current lines driven between the respective electrodes. transfer impedance value $Z_{T}$ as well as the contribution $Z_{b}$ of the background volume outside the stomach region and the contribution $Z_{s t}$ of the stomach volume were determined for each of the measurements (TPIM1, TPIM2, FIM) using the following equations:

$$
\begin{aligned}
& Z_{b}=\int_{b} \rho_{b} S d V \\
& Z_{s t}=\int_{s t} \rho_{s t} S d V \\
& Z_{T}=Z_{b}+Z_{s t}
\end{aligned}
$$

where $\rho_{b}$ is the resistivity of the background of the trunk volume outside the stomach, assumed uniform throughout, $\rho_{s t}$ is the resistivity of the stomach region, assumed uniform, and $S$ is the point sensitivity as mentioned before in Equation 1. The contribution of the stomach to the total transfer impedance was expressed as,

$$
C_{s t}=\frac{Z_{s t}}{Z_{T}} \times 100 \%
$$

Obviously, the target would be to make $C_{s t}$ as large as possible. The simulations were performed to obtain $C_{s t}$ for the following two situations.

Simulation A. The whole volume conductor, including the stomach, has the same conductivity, that of isotonic $0.9 \%$ saline $(\sigma=1.2 \mathrm{~S} / \mathrm{m}$, relative permittivity, $\varepsilon=80)$, i.e., $\rho_{s t}=\rho_{b}$.

Simulation B. The stomach has a higher conductivity, equal to that of $3.5 \%$ saline $(\sigma=5 \mathrm{~s} / \mathrm{m}$, relative permittivity, $\varepsilon=80$ ), while the rest of the trunk has the conductivity of that of isotonic $0.9 \%$ saline.

For both, the conductivity and relative permittivity of the part of the electrodes outside the volume conductor was taken as $6 \times 10^{7} \mathrm{~S} / \mathrm{m}$ and 1 , respectively. As mentioned before, the part of the electrode falling inside the volume conductor was made to have the same electrical parameters as for the background volume of the trunk.

A frequency of $200 \mathrm{kHz}$ was assumed for the simulation. The electrical parameters used are summarized in Table 1. The geometrical parameters appropriate for the chosen model are shown in Table 2 . The geometries of the electrode placement configurations are given together with the calculated values of $C_{s t}$ in the results section.

Table 1: Assumed electrical values for simulation. Frequency $=200 \mathrm{kHz}$. Current $=1 \mathrm{~A}$

\begin{tabular}{cccc}
\hline Electrical Properties & $\begin{array}{c}\text { Background } \\
\text { trunk volume* }\end{array}$ & $\begin{array}{c}\text { Stomach } \\
\text { volume** }\end{array}$ & $\begin{array}{c}\text { Electrode } \\
\text { (copper) }\end{array}$ \\
\hline $\begin{array}{c}\text { Conductivity }(\sigma) \\
\text { [S/m] }\end{array}$ & 1.2 & 5 & $6 \times 10^{7}$ \\
$\begin{array}{c}\text { Relative Permittivity } \\
(\epsilon)\end{array}$ & 80 & 80 & 1 \\
\hline
\end{tabular}

* Equivalent to that of $0.9 \%$ saline at $20^{\circ} \mathrm{C}$, also for stomach in Simulation A.

** Equivalent to that of $3.5 \%$ Saline (sea water) at $20^{\circ} \mathrm{C}$, for simulation B. 
While performing the analyses, it was found out that the initial trial configuration of TPIM2 has a lower $C_{s t}$ value compared to that for TPIM1. It was suggested that the electrode placement need some further changes. At this point, further guidance was taken from an equipotential concept [15], that region bounded by the equipotentials passing through the potential electrodes have a high contribution to the transfer impedance. Based on this concept the position of the electrodes for both TPIM1 and TPIM2 were examined qualitatively with the help of Figure 5.

For TPIM1, the equipotential lines bound the stomach region well on the top and bottom. On the other hand, the equipotential lines for Trial-TPIM2 include a large amount of background volume on the left side of the stomach.

Table 2: Geometrical parameters appropriate for the chosen model

\begin{tabular}{lr}
\hline Volume of whole Trunk (including stomach) & $28430 \mathrm{~cm}^{3}$ \\
Volume of Stomach & $1293 \mathrm{~cm}^{3}$ \\
Stomach volume with respect to whole volume & $4.54 \%$ \\
Horizontal Cross Sectional Area of Trunk & $711.4 \mathrm{~cm}^{2}$ \\
Horizontal Cross Sectional Area of Stomach & $161.4 \mathrm{~cm}^{2}$ \\
Area of Stomach with respect to Area of Trunk & $22.69 \%$ \\
(cross-sectional) & \\
\hline
\end{tabular}

Therefore, based on this pictorial information, the lower potential electrode $V_{2}^{\prime}$ was shifted to $V_{2 N}{ }^{\prime}$ as shown for TPIM2 so that the stomach is bounded well between the two equipotential lines. Consequently, it was envisaged that TPIM2 will have a higher contribution from the stomach so that the resulting FIM will also have a higher contribution from the stomach. This was actually observed, and so all the analyses shown in this work use TPIM2, not the Trial-TPIM2.

In order to have an insight into the effect of the separation between the adjacent current and voltage electrodes, the electrode separations near to the stomach $\left(I_{1}-V_{1}\right.$ and $\left.I_{2}-V_{2}\right)$ were increased or decreased by about $20 \%$ from the intuitively chosen ones and the results were compared to see which separation gives the maximum contribution from the stomach.

The electrode configuration giving the maximum contribution of the stomach was used to obtain color maps of the point sensitivity in the horizontal plane at the vertical midpoint of the stomach, which is also the plane of the centers of the electrodes. These color maps provide further insight into the contribution of the different regions to the total transfer impedance, which is very helpful (not shown).

\section{Strip electrodes}

Small electrodes, as used in the above work have wide diverging current patterns within the volume conductor. Since the stomach has a vertical height, in order to get more contribution from this 3D stomach, long strip electrodes, as suggested by Rabbani [14] was also used for the electrode configuration giving the maximum contribution of the stomach using the small cylindrical electrodes $(2 \mathrm{~cm}$ diameter). The comparison would give valuable insight. The strip electrodes were rectangular, $2 \mathrm{~cm} \times 2 \mathrm{~cm}$ in cross section, and of variable lengths: $5 \mathrm{~cm}, 6 \mathrm{~cm}$ and $8 \mathrm{~cm}$. These were positioned centrally on the stomach in the vertical direction. In this case, the current paths are expected to diverge less within the vertical extension of the stomach region giving higher current densities in the stomach contributing to a higher contribution in the total transfer impedance.

\section{A new electrode configuration, orthogonal concentric TPIMs in the vertical plane}

Another electrode configuration was suggested and tested during the present work. In this configuration, the TPIM1 of the previous configuration was used in the horizontal plane while a similar configuration of electrodes, TPIM1-O was placed orthogonal to TPIM1 as shown in Figure 6. Since the

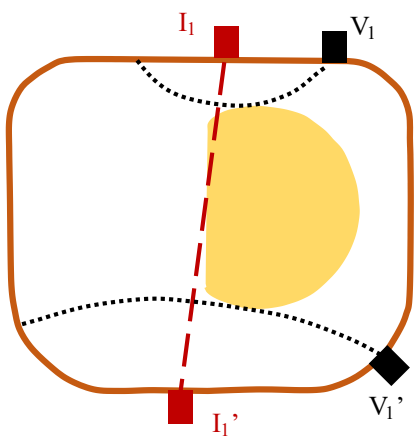

TPIM1

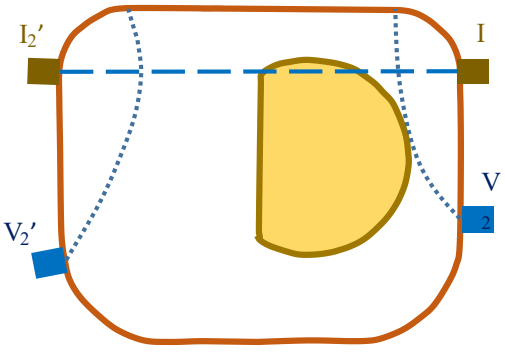

Trial-TPIM2

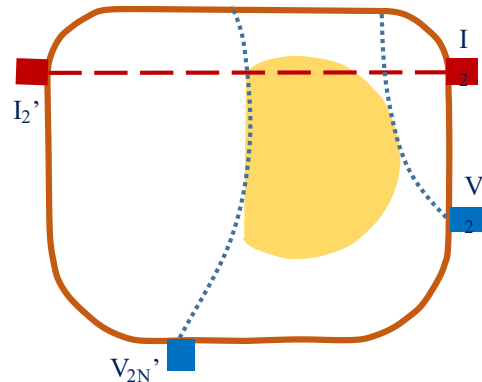

TPIM2

Fig 5: Visualization of electrode placement based on equipotential concept for both TPIM1 and TPIM2. Dashed lines show the maximum current lines through the current-drive electrodes. Dotted lines show approximate shape of equipotential lines through the potential electrodes due to the respective currents. For TPIM1, the equipotential lines bound the stomach region well. The equipotential lines for the electrode configuration for Trial-TPIM2 includes a large amount of background space on the left side of the stomach. For the new configuration of TPIM2, the lower potential electrode $V_{2}{ }^{\prime}$ has been shifted to $V_{2 N}{ }^{\prime}$ based on equipotential visualization so that the stomach is bounded well between the two equipotential lines. This results in an improvement. 


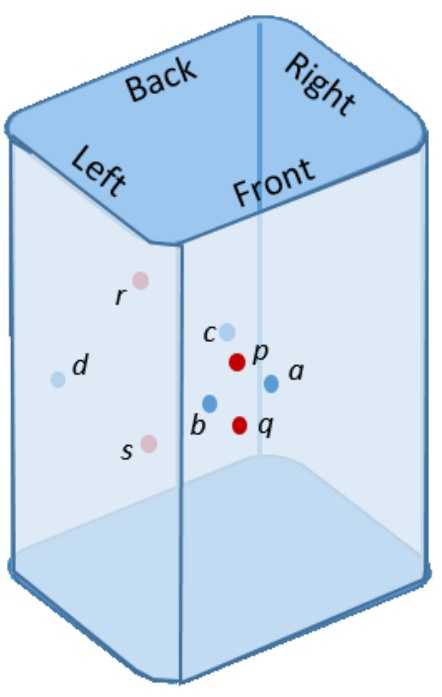

Fig 6. Orthogonal concentric TPIMs in the vertical plane.

stomach is nearer to the front surface, to show the nearside electrodes, $\mathrm{I}_{1}-\mathrm{V}_{1}$ and the orthogonal ones, this diagram reverses the geometrical representation shown in Figure 2 earlier. Here, $a, b$ indicate the electrodes $I_{1}-V_{1}$ in the horizontal plane for TPIM1 as before and $p, q$ indicate the electrodes for TPIM1-O in the vertical plane on the front surface. Corresponding electrodes on the back surface are shown faded in the diagram, indicated by $c$, $d$ and $r, s$ respectively. Essentially this configuration is expected to have high sensitivity in a solid angle through the stomach.

All the results and the comparative analyses are presented in the results section below.

\section{Ethical approval}

The conducted research is not related to either human or animal use.

\section{Results and observations}

For the geometry, the origin $(0,0,0)$ has been taken at the lower left corner of the trunk model as shown in Figure 2, which is on the right side of the back of the trunk, in physical perspective. The co-ordinate $(x, y, z)$ of the center of the circular cylinder used to make the stomach model is $(19,14$, 19 ) in $\mathrm{cm}$, as can be seen from Figures 2 and 3 . The $z-$ coordinate for all electrodes were also chosen at $19 \mathrm{~cm}$, the vertical central plane of the stomach. The reference points of the cylindrical electrodes are automatically chosen by COMSOL and these are shown in Figure 7.

For the long vertical strip electrodes used in one of the studies, the center of the strips were located at $z=19 \mathrm{~cm}$, while the $x$ and $y$ coordinates are as referenced in Figure 7. Separations between pairs of electrodes were calculated as the direct straight path length between the mid-points of these electrodes touching the trunk volume, not based on their geometrical reference points.

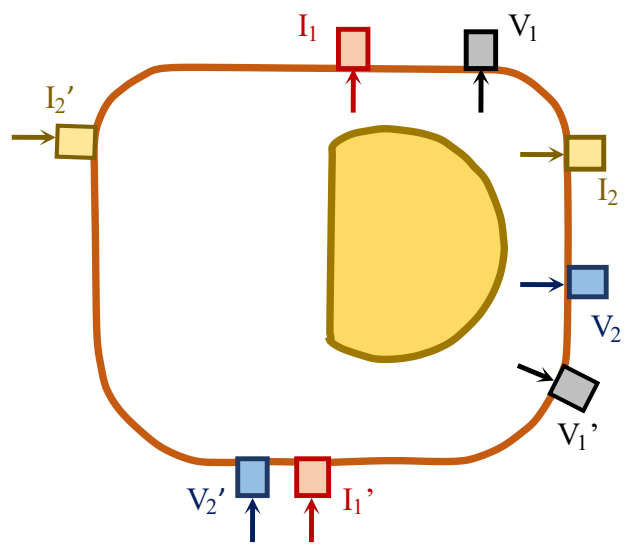

Fig 7. Geometrical reference points of electrodes, shown by arrows.

The results of the various simulations performed using COMSOL are given below, together with brief observations wherever feasible. The names of the electrodes are also as in Figure 7. The coordinates of the electrodes are also given in the tables, where necessary. The values in scientific notation follows that of EXCEL where $1.0 \mathrm{E} 6$ would mean $1 \times 10^{6}$.

Table 3: Simulation-A for TPIM1 for varying separation of $I_{1}-V_{1}$ Electrode Co-ordinates for 3 separations: [I1:(16,25,19), V1:(20,25,19)]; [I1:(14,25,19), V1:(22,25,19)]; [I1:(12,25,19), V1:(24,25,19)]. Fixed $\left[11^{\prime}=(16,-3,19) ; V^{\prime}=(29,6.5,19)\right]$

Direct straight-line separation for $\mathrm{I}_{1}{ }^{\prime}-\mathrm{V}_{1}{ }^{\prime}=15.4 \mathrm{~cm}$

\begin{tabular}{cccc}
\hline & \multicolumn{3}{c}{ Separation of $\mathrm{I}_{\mathbf{1}}-\mathrm{V}_{\mathbf{1}}, \mathbf{c m}$} \\
Simulated parameters & 4 & 8 & 12 \\
\hline Maximum Sensitivity* & $7.71 \mathrm{E}+06$ & $1.27 \mathrm{E}+06$ & $8.99 \mathrm{E}+05$ \\
Minimum Sensitivity* & $-2.22 \mathrm{E}+06$ & $-3.66 \mathrm{E}+05$ & $-2.36 \mathrm{E}+05$ \\
$\begin{array}{c}\text { Total Impedance (ohm) } \\
\text { Stomach Impedance } \\
\text { (ohm) }\end{array}$ & 3.23 & 1.57 & 1.07 \\
\hline $\begin{array}{c}\text { \% Contribution of } \\
\text { stomach }\end{array}$ & 0.61 & 0.35 & 0.23 \\
\hline
\end{tabular}

* values on the electrode plane ( $x-y$ plane) only

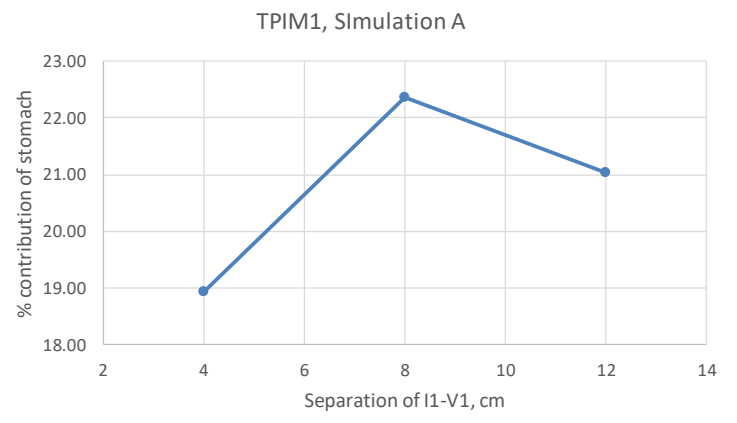

Fig 8. Percentage Contribution of stomach in Simulation-A for TPIM1 for varying separation of $\mathrm{I}_{1}-\mathrm{V}_{1}$ 
Table 4: Simulation-A for TPIM2 for varying separation of $I_{2}-V_{2}$ Electrode Co-ordinates for 3 separations: [12:( 30,14,19), V2: $(30,18,19)] ;[12:(30,12,19), \mathrm{V} 2:(30,20,19)] ;[12:(30,10,19)$, V2: $(30,22,19)]$. Fixed $\left[11^{\prime}=(13,-3,19), V_{1}^{\prime}=(2,21,19)\right]$

Direct straight-line separation for $\mathrm{I}_{2}{ }^{\prime}-\mathrm{V}_{2}{ }^{\prime}=24.7 \mathrm{~cm}$

\begin{tabular}{cccc}
\hline & \multicolumn{3}{c}{$\mathbf{I}_{\mathbf{2}}-\mathbf{V}_{\mathbf{2}}, \mathbf{c m}$} \\
Simulated parameters & 4 & 8 & 12 \\
\hline Max Sensitivity* & $9.65 \mathrm{E}+05$ & $5.10 \mathrm{E}+05$ & $4.01 \mathrm{E}+05$ \\
Min Sensitivity* & $-1.35 \mathrm{E}+07$ & $-2.99 \mathrm{E}+06$ & $-1.14 \mathrm{E}+06$ \\
Total Impedance (ohm) & 3.08 & 1.63 & 1.06 \\
Stomach Impedance (ohm) & 0.60 & 0.38 & 0.21 \\
\hline \% Contribution of stomach & $\mathbf{1 9 . 5 3}$ & $\mathbf{2 3 . 0 7}$ & $\mathbf{2 0 . 2 2}$ \\
\hline
\end{tabular}

* values on the electrode plane ( $\mathrm{x}$-y plane) only

Table 3 shows the simulated values obtained for TPIM1 with changing the separation of electrodes $I_{1}-V_{1}$, nearest to the stomach on the front side. The separation of the other electrodes $I_{1}{ }^{\prime}-V_{1}{ }^{\prime}$ remain the same at $15.4 \mathrm{~cm}$. This is the result for Simulation-A as mentioned before, with the stomach and the background having the same electrical parameters as that for isotonic $(0.9 \%)$ saline. The maximum and minimum sensitivity shown in the table refers to values on the electrode plane ( $x$-y plane) only, while the impedance values refer to the whole volume of the trunk or the stomach as appropriate. The percentage contribution of the stomach for the three separations are shown in the table as well as in Figure 8 .

Table 5: Simulation-A for FIM with best TPIM configurations Direct straight-line separations: I1-V1 $=8 \mathrm{~cm}, \mid 1^{\prime}-\mathrm{V} 1{ }^{\prime}=15.4 \mathrm{~cm}$, I2-V2 = $8 \mathrm{~cm}, 12^{\prime}-\mathrm{V}^{\prime}{ }^{\prime}=24.7 \mathrm{~cm}$

\begin{tabular}{llll}
\hline Simulated parameters & TPIM1 & TPIM2 & FIM \\
Max Sensitivity* & $1.27 \mathrm{E}+06$ & $5.10 \mathrm{E}+05$ & $6.40 \mathrm{E}+05$ \\
Min Sensitivity* & $-3.66 \mathrm{E}+05$ & $-2.99 \mathrm{E}+06$ & $-1.48 \mathrm{E}+06$ \\
Total Impedance (ohm) & 1.57 & 1.63 & 1.60 \\
Stomach Impedance (ohm) & 0.35 & 0.38 & 0.36 \\
\hline \% Contribution of stomach & $\mathbf{2 2 . 3 6}$ & $\mathbf{2 3 . 0 7}$ & $\mathbf{2 2 . 7 3}$ \\
\hline
\end{tabular}

*values on the electrode plane ( $x$-y plane) only

Table 4 shows the simulated values (Simulation-A) obtained for TPIM2 with changing the separation of electrodes $I_{2}-V_{2}$, nearest to the stomach on the left side. The separation of the other electrodes $\mathrm{I}_{2}{ }^{\prime}-\mathrm{V}_{2}{ }^{\prime}$ remain the same at $24.7 \mathrm{~cm}$. The percentage contribution of the stomach for the three separations are shown in the table as well as in Figure 9.

It is interesting to note that for both TPIM1 and TPIM2, the separations of the $I$ and $V$ electrodes nearest to the stomach giving the maximum $C_{s t}$ values were the ones that were initially chosen based on qualitative visualisations based on point sensitivity considerations and equipotentials. Increase and decrease of the separation led to reduced $C_{s t}$ values. Table 5 gives a comparison of the best $C_{s t}$ values obtained using TPIM1 and TPIM2 and FIM using these two electrode configurations. Figure 10 shows this result through a histogram.

Table 6: Simulation-B for FIM with best TPIM configurations

Direct straight-line separations: $|1-V 1=8 \mathrm{~cm},| 1^{\prime}-\mathrm{V} 1^{\prime}=15.4 \mathrm{~cm}$, I2-V2 = $8 \mathrm{~cm}, 12^{\prime}-\mathrm{V}^{\prime}{ }^{\prime}=24.7 \mathrm{~cm}$

\begin{tabular}{llll}
\hline Simulated parameters & TPIM1 & TPIM2 & FIM \\
\hline Max Sensitivity* & $8.41 \mathrm{E}+05$ & $4.86 \mathrm{E}+05$ & $4.23 \mathrm{E}+05$ \\
Min Sensitivity* & $-2.41 \mathrm{E}+05$ & $-2.09 \mathrm{E}+06$ & $-1.05 \mathrm{E}+06$ \\
Total Impedance (ohm) & 1.01 & 1.096 & 1.055 \\
Stomach Impedance (ohm) & 0.389 & 0.333 & 0.3611 \\
\hline \% Contribution of stomach & $\mathbf{3 8 . 5 1}$ & $\mathbf{3 0 . 3 8}$ & $\mathbf{3 4 . 2 3}$ \\
\hline
\end{tabular}

*values on the electrode plane ( $x-y$ plane) only

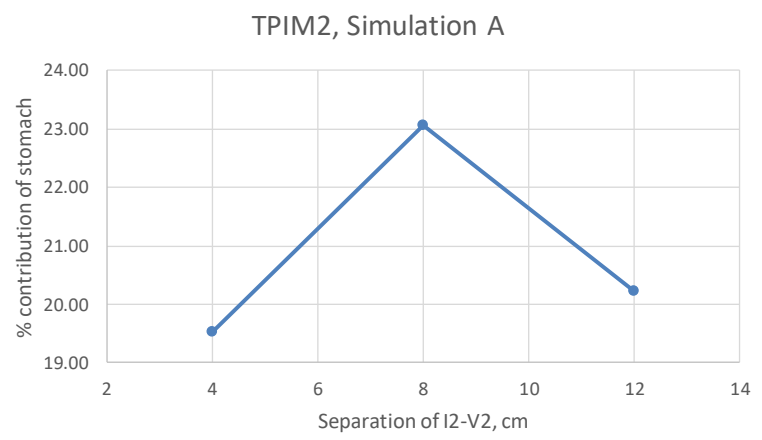

Fig 9. Percentage Contribution of stomach in Simulation-A for TPIM2 for varying separation of $\mathrm{I}_{2}-\mathrm{V}_{2}$

\section{Comparison for Simulation A}

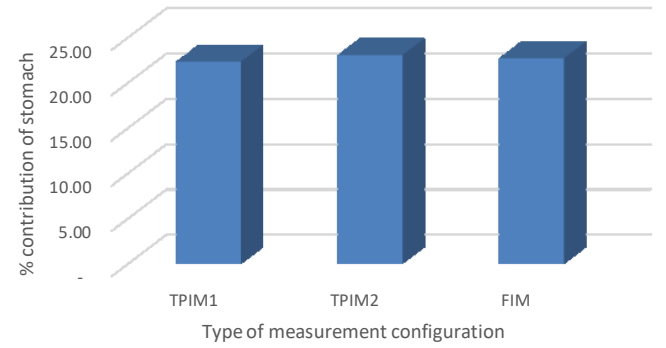

Fig 10. Percentage Contribution of stomach in Simulation-A for TPIM1, TPIM2 and FIM using the best combination of electrode configurations

\section{Comparison for Simulation B}

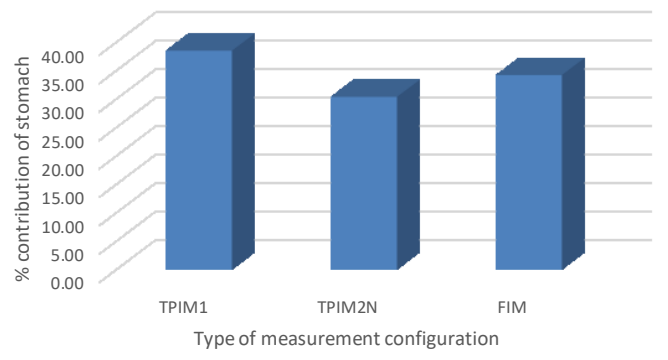

Fig 11. Percentage Contribution of stomach in Simulation-B for TPIM1, TPIM2 and FIM using the best combination of electrode configurations. 
Table 7: Simulation-A for Strip Electrodes of different lengths Strip electrode cross section: $2 \mathrm{~cm} \times 2 \mathrm{~cm}$

Direct straight-line separations: $11-\mathrm{V} 1=8 \mathrm{~cm}, 11^{\prime}-\mathrm{V} 1^{\prime}=15.4 \mathrm{~cm}, 12-\mathrm{V} 2=8 \mathrm{~cm}, 12^{\prime}-\mathrm{V} 2^{\prime}=24.7 \mathrm{~cm}$

\begin{tabular}{|c|c|c|c|c|c|c|c|c|c|}
\hline \multirow[b]{2}{*}{$\begin{array}{l}\text { Simulated } \\
\text { Parameters }\end{array}$} & \multicolumn{3}{|c|}{ Length $=5 \mathrm{~cm}$} & \multicolumn{3}{|c|}{ Length $=6 \mathrm{~cm}$} & \multicolumn{3}{|c|}{ Length $=8 \mathrm{~cm}$} \\
\hline & TPIM1 & TPIM2 & FIM & TPIM1 & TPIM2 & FIM & TPIM1 & TPIM2 & FIM \\
\hline Max Sensitivity* & $3.90 \mathrm{E}+04$ & $4.47 E+04$ & $2.43 E+04$ & $2.64 \mathrm{E}+04$ & $2.37 E+04$ & $1.67 \mathrm{E}+04$ & $5.42 E+04$ & $3.05 E+04$ & $3.27 E+04$ \\
\hline Min Sensitivity* & $-6.32 E+04$ & $-7.53 E+04$ & $-3.60 E+04$ & $-5.14 E+04$ & $-6.73 E+04$ & $-3.26 E+04$ & $-5.77 E+04$ & $-1.57 E+05$ & $-7.80 \mathrm{E}+04$ \\
\hline $\begin{array}{c}\text { Total } \\
\text { Impedance, } \Omega\end{array}$ & 1.49 & 1.57 & 1.53 & 1.40 & 1.48 & 1.44 & 1.47 & 1.55 & 1.51 \\
\hline $\begin{array}{c}\text { Stomach } \\
\text { Impedance, } \Omega\end{array}$ & 0.33 & 0.36 & 0.34 & 0.29 & 0.32 & 0.30 & 0.32 & 0.35 & 0.33 \\
\hline $\begin{array}{c}\text { \% Contribution } \\
\text { of stomach }\end{array}$ & 21.82 & 22.55 & 22.23 & 20.82 & 21.49 & 21.16 & 21.57 & 22.33 & 21.93 \\
\hline
\end{tabular}

*values on the $x-y$ plane at the vertical center of the electrode and stomach

Table 6 gives the results of Simulation-B in which the stomach is assumed to have a higher conductivity, equivalent to that of $3.5 \%$ saline (sea water) using the same electrode configurations as for Table 5 . The rest of the trunk volume is assumed to have the electrical parameters appropriate for isotonic (0.9\%) saline. Figure 11 shows this result through a histogram. It is interesting to note that although the $C_{s t}$ values are almost similar for all the three measurements for Simulation-A, TPIM1 attains a higher value than TPIM2 for Simulation $B$, with FIM being inbetween.

Table 7 shows Simulation-A values obtained for all the three configurations for different lengths of rectangular strip electrodes, for the chosen best placements of electrodes and Figure 12 shows the results graphically.

Table 8. Simulation-A for Orthogonal Electrode I1-V1 = $8 \mathrm{~cm}, I^{\prime}-V_{1}{ }^{\prime}=15.4 \mathrm{~cm}, I 2-V 2=8 \mathrm{~cm}, I^{\prime}-\mathrm{V} 2{ }^{\prime}=15.4 \mathrm{~cm}$

\begin{tabular}{cccc}
\hline Simulated parameter & TPIM1 & TPIM1-O & FIM \\
\hline Total Impedance (ohm) & 1.58 & 1.40 & 1.49 \\
Stomach Impedance (ohm) & 0.35 & 0.29 & 0.32 \\
\hline \% Contribution of stomach & $\mathbf{2 2 . 3 4}$ & $\mathbf{2 0 . 9 0}$ & $\mathbf{2 1 . 6 7}$ \\
\hline
\end{tabular}

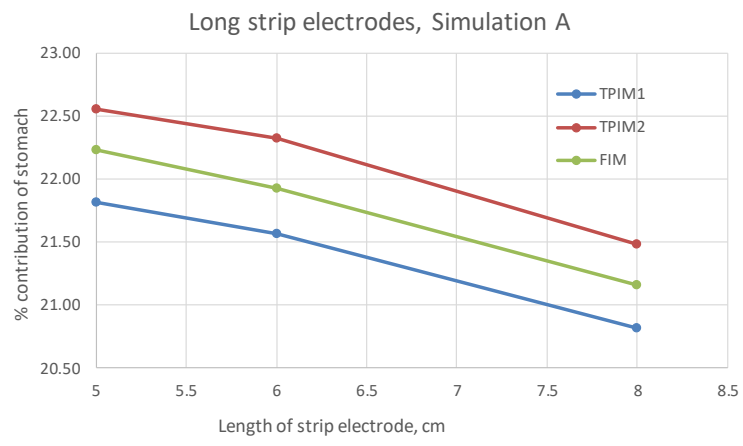

Fig 12. Percentage Contribution of stomach in Simulation-A for long strip electrodes of different lengths, for all the three configurations, TPIM1, TPIM2 and FIM using the electrode placements used for the small round electrodes.

Figure 13 shows percentage contribution of stomach in Simulation-A for $2 \mathrm{~cm}$ round electrodes and for $5 \mathrm{~cm}$ long strip electrodes of $2 \mathrm{~cm} \times 2 \mathrm{~cm}$ cross section, for all the three configurations, TPIM1, TPIM2 and FIM.

Table 8 shows the impedance values for the newly proposed orthogonal configuration, targeting a solid angle into the trunk volume around the stomach. All the three give reasonably good contribution for the stomach.

\section{Comparison, round \& strip electrodes}

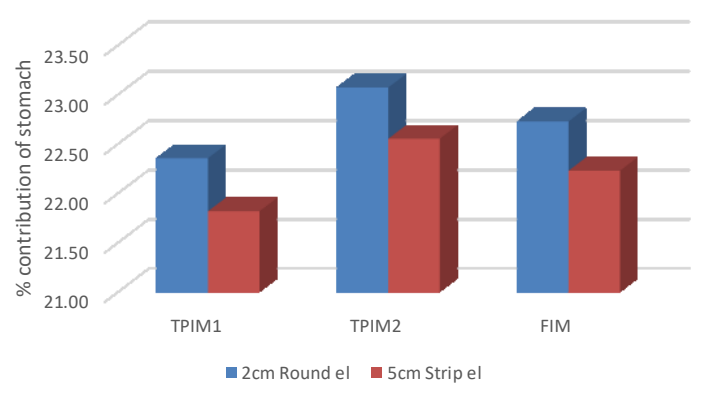

Fig 13. Percentage Contribution of stomach in Simulation-A for $2 \mathrm{~cm}$ round electrodes and for $5 \mathrm{~cm}$ long strip electrodes of $2 \mathrm{~cm} \times 2 \mathrm{~cm}$ cross section, for all the three configurations, TPIM1, TPIM2 and FIM.

\section{Discussion}

For any electrical impedance measurement probing deep inside a body, the popular way would be using EIT. However, the main motivation of the authors' group is to develop technologies for the low income countries, which should be simple and robust. Therefore, if simple TPIM or FIM can be used to get information on deep organs with reasonable sensitivity, it may find a useful application, not only for the low income countries, but for the high income countries as well.

The success of using a TPIM or FIM depends on judicial placement of electrodes, where understanding of the basic visualization based on point sensitivity equation and equipotential concepts, both are necessary. This has been illustrated through this work.

The present work has tried small round electrodes and long strip electrodes. Apparently they all gave percentage contribution of the stomach of a similar order, with no 
preference for either. This suggests that using a small round electrode is preferable in view of practical considerations.

Almost all the methods give a percentage contribution of the stomach a little above $20 \%$. It is interesting to note that the percentage of cross sectional area of the modeled stomach to that of the whole trunk is of similar order (22.7\%). Therefore, the values obtained are possibly the maximum that one can get through TPIM or FIM.

From the similar results for TPIM and FIM, one might argue that since TPIM is simpler, it should be preferable. However, it should be recognized that TPIM has more contribution from negative sensitivity zones, which cancel to some extent in FIM [11]. The maximum and minimum sensitivities presented in the tables indicate that these are slightly less in FIM in general. However, these values only pertain to the electrode plane. Usually, these values are expected to be less in 3D.

If the changes only occur in the stomach, then either TPIM or FIM may give useful results. However, if other organs like the duodenum and the intestine change, which is very likely, these changes will affect the result. Therefore, FIM may have an advantage here in reducing the effects of nearby organs. Further studies taking such organs in consideration should be taken up which will show which method is best suited for a particular application.

Therefore, through further studies following the approach presented in this paper, one may get useful physiological and diagnostic information in the deep organs like the stomach using simple techniques like TPIM or FIM.

\section{Conflict of interest}

Authors state no conflict of interest.

\section{Acknowledgements}

The authors acknowledge financial support provided by the International Science Programme (ISP) of Uppsala University, Sweden for this work. One of the authors (RH) acknowledges the scholarship offered through Marie Curie Scholarship, under a gender balance programme of ISP at Dhaka University.

\section{References}

1. Nyboer J. Electrical Impedance Plethysmography: The electrical resistive measure of the blood pulse volume, peripheral and central blood flow. Charles. C. Thomas; 1959. https://doi.org/10.1016/0002-8703(60)90473-7

2. Martinsen $\varnothing \mathrm{G}$, Grimnes $S$. The concept of transfer impedance in bioimpedance measurements. In 4th European Conference of the International Federation for Medical and Biological Engineering 2009 (pp. 1078-1079). Springer, Berlin, Heidelberg. https://doi.org/10.1007/978-3-540-89208-3_257

3. Lukaski HC, Johnson PE, Bolonchuk WW and Lykken GI. Assessment of fat-free mass using bioelectrical impedance measurements of the human body. Am J Clin Nutr. 1985; 41(4):810-817. https://doi.org/10.1093/ajcn/41.4.810
4. Kushner RF. Schoeller DA. 1986. Estimation of total body water by bioelectrical impedance analysis, The American Journal of Clinical Nutrition, 1986; 44(3):417-424. https://doi.org/10.1093/ajcn/44.3.417

5. Henderson RP, Webster JG. An impedance camera for spatially specific measurements of the thorax. IEEE Trans Biomed Eng. 1978; 3:250-254.

https://doi.org/10.1109/tbme.1978.326329

6. Barber DC, Brown BH, Freeston IL. Imaging spatial distributions of resistivity using applied potential tomography. Electron. Lett. 1983; 19(22):933-935. https://doi.org/10.1049/el:19830637

7. Rabbani KS and Kabir H. Studies on the effect of the third dimension on a two dimensional Electrical Impedance Tomography system, Clin Phy \& Physiol Meas. 1991; 12(4):393-402. https://doi.org/10.1088/0143-0815/12/4/009

8. González G, Huttunen JMJ, Kolehmainen V, Seppänen A and Vauhkonen, M. 2016. Experimental evaluation of 3D electrical impedance tomography with total variation prior. Inverse Problems in Science and Engineering. 2016; 24(8):1411-1431. https://doi.org/10.1080/17415977.2015.1113961

9. Rabbani KS, Sarker M, Akond MH, Akter T. Focused impedance measurement (FIM): a new technique with improved zone localization. Annals of the New York Academy of Sciences. 1999 Apr; 873(1):408-420. https://doi.org/10.1111/j.1749-6632.1999.tb09490.x

10. Rabbani KS, Karal MA. A new four-electrode Focused Impedance Measurement (FIM) system for physiological study. Annals of biomedical engineering. 2008; 36(6):107277. https://doi.org/10.1007/s10439-008-9470-7

11. Rabbani KS. Focused Impedance Method: Basics and Applications. In: Simini F., Bertemes-Filho P. (eds) Bioimpedance in Biomedical Applications and Research. Springer, Cham; 2018 pp. 137-185. https://doi.org/10.1007/978-3-319-74388-2_9.

12. Islam N, Rabbani KS, Wilson AJ. 2010. The sensitivity of focused electrical impedance measurements, Physiol. Meas. 2010; 31:S97-S109. https://doi.org/10.1088/0967-3334/31/8/s08

13. Kadir, MA, Baig, TN, Rabbani, KS. Focused impedance method to detect localized lung ventilation disorders in combination with conventional spirometry. Biomed. Eng.: Appl. Basis Commun. 2015; 27(03):1550029. https://doi.org/10.4015/s1016237215500295

14. Rabbani KS. Simple electrode configurations for probing deep organs using Electrical Bio-Impedance techniques. Bangladesh Journal of Medical Physics. 2018; 11(1):1-15. https://doi.org/10.3329/bjmp.v11i1.44053

15. Baker LE. Principles of the impedance technique. IEEE Engineering in Medicine and Biology Magazine. 1989; 8(1):1115.

16. Grimnes $S$ and Martinsen $\varnothing \mathrm{G}$. Bioelectricity and Biompedance Basics, p.188, Elsevier; 2015. 
17. Roy SK, Karal MA, Kadir MA, Rabbani KS. A new six-electrode electrical impedance technique for probing deep organs in the human body. Eur. Biophys. J. 2019; 48(8):711-719. https://doi.org/10.1007/s00249-019-01396-x

18. Comsol Multiphysics, 2013. AC/DC Module user's guide. Accessed: 6 December, 2019. https://www.comsol.com/acdc-module

19. University of Texas Medical Branh information, Accessed: 6 December, 2019.

http://ar.utmb.edu/webpath/radiol/radnorm/abct11.htm
20. Sauerheber R, Heinz B. Temperature effects on conductivity of seawater and physiologic saline, Mechanism and Significance. J. Chem. Sci. 2015; 6(4):1000109.

21. Peyman A, Gabriel C, Grant EH. Complex permittivity of sodium chloride solutions at microwave frequencies. Bioelectromagnetics. 2007; 28(4):264-274. https://doi.org/10.1002/bem.20271

22. Geselowitz DB. An application of electrocardiographic lead theory to impedance plethysmography. IEEE Trans. Biomed. Eng. 1971; 18:38-41. https://doi.org/10.1109/tbme.1971.4502787 